\title{
Ceramic Stereolithography: Additive Manufacturing for 3D Complex Ceramic Structures
}

\author{
Chang-Jun Bae ${ }^{\dagger}$, Arathi Ramachandran, Kyeongwoon Chung, and Sujin Park \\ Process Innovation Department, Korea Institute of Materials Science, Changwon 51508 Korea \\ (Received October 10, 2017; Revised November 16, 2017; Accepted November 16, 2017)
}

\begin{abstract}
Ceramic processing to fabricate 3D complex ceramic structures is crucial for structural, energy, environmental, and biomedical applications. A unique process is ceramic stereolithography, which builds ceramic green objects from CAD files from many thin liquid layers of powder in monomer, which are solidified by polymerization with a UV laser, thereby "writing" the design for each slice. This approach directly writes layers in liquid ceramic suspension and allows one to fabricate ceramic parts and products having more accurate, complex geometries and smooth surfaces. In this paper, both UV curable materials and processes are presented. We focus on the basic material principles associated with free radical polymerization and rheological behavior, cure depth and broadening of cured lines, scattering at ceramic interface and their corresponding simulation. The immediate potentials for ceramic AM to change industry fabrication are also highlighted.
\end{abstract}

Key words : Additive manufacturing, Ceramic 3D printing, Rheology, Light scattering, Internal stress

\section{Introduction}

$\mathrm{N}$ ew ceramic processing for fabricating 3D ceramic structures will provide huge opportunities in areas of structural, energy, environmental, and biological applications, due to the superior physical and chemical properties of ceramics, such as their low thermal expansion coefficient and excellent wear-, and corrosion-resistance. ${ }^{1-3)}$ Conventional subtractive manufacturing (SM) fabricates products by removing materials from a large stock and is expensive, difficult, and time consuming to apply to fabricating complex ceramic structures out of hard and brittle ceramics. ${ }^{4-5)}$ New methods to effectively sculpture ceramics should be developed to meet new market demands.

Contrary to the conventional subtractive methods, new ceramic additive manufacturing (AM) creates $2 \mathrm{D}$ layers in $\mathrm{X}-\mathrm{Y}$ planes and directly fabricates $3 \mathrm{D}$ structures from $\mathrm{CAD}$ data using layer-by-layer processes, mitigating the hardness and brittleness of ceramics and directly creating final 3D ceramic objects. ${ }^{6}$ The ceramic AM technologies are also known among both engineering and scientific communities as solid freeform fabrication (SFF), a term coined in the late 1980s, and rapid manufacturing or rapid prototyping (RP), a term that was popularized in $2000 .^{7-9)}$ According to the states of the materials, ceramic AM can be categorized into solid state ceramic powders and liquid state ceramic suspension.

Solid state ceramic powders are mainly used in the

\footnotetext{
Corresponding author: Chang-Jun Bae

E-mail : baecj01@kims.re.kr

Tel : +82-55-280-3251 Fax : +82-55-280-3289
}

ceramic 3D printing (3DP) or selective laser sintering (SLS), where 3DP nozzles ejects glue onto the ceramic powders or SLS expose UV laser onto binder coated ceramic powders to form $2 \mathrm{D}$ layers, respectively. ${ }^{10,11)}$ Liquid state ceramic suspensions are operated in the ceramic stereolithography (CerSLA) or digital light process (DLP), where a UV laser or digital micro-mirror solidifies thin liquid layers of powder in polymer monomer to fabricate ceramic green objects. ${ }^{12,13)}$ The ability to directly write layers in liquid ceramic suspension guarantees that CerSLA or DLP is capable of fabricating ceramic parts and products having more accurate, complex geometries and smooth surfaces. ${ }^{14)}$

Our review article on ceramic additive manufacturing is focusing on liquid state ceramic suspensions which have various advantages of accuracy and surface finish than solid state ceramic powders as explained above. In Section II, we provide a brief overview of the basic material principles associated with free radical polymerization, photo-curable monomers, and viscosity of ceramic suspensions. The success of liquid type ceramic AM relies on how to increase ceramic powder loading in green bodies at minimal increases of the suspension viscosity for fabricating high quality, dense, defect-free ceramics. Section III describes representative cure depth and broadening of cured lines, scattering at ceramic interface and their corresponding simulation, and build styles and post processes used to make 3D complex ceramic structures. Analytic examples highlighted in each section provide physical meaning and facilitate direct comparisons of various ceramic suspensions. Finally, we have provided two examples where we see an immediate potential for ceramic AM to change industry fabrication in section IV. 


\section{Fundamentals of UV Curable Materials}

\subsection{Free radical polymerization}

Solvent-free, fast, and room temperature operative, photopolymerization reactions are used in a wide variety of applications from coatings to electronics and 3D printing. Light based, ceramic 3D printing formulations consist of photoinitiators, photocurable monomerss (and/or oligomers), and ceramic powder. When the monomer, photoinitiator, ceramic powder slurries are illuminated, the photoinitiator generates active radical or cation species that attack double bonds in the monomer to initiate polymerization as shown in Fig. 1. Subsequent reactions between active chain end and monomer makes polymer chains grow until they lose their activity by termination processes such as coupling between two radicals. The polymer chain continues to extend until a termination reaction occurs. If monomer have more than one functional group which can participate in polymerization, cross-linked network of polymer chains is generated rather than linear chain. In light-induced ceramic 3D printing, the polymer network generated by photopolymerization holds ceramic particles in its matrix to shape up 3D structure of the green body. The green body undergoes burn-out process for removal of organic components, followed by sintering to form dense ceramic body.

There are two representative photopolymerization mechanisms: radical polymerization and cationic polymerization. In cationic polymerization, onium salts such as triarylsulfonium salt are generally used as an initiator to form $\mathrm{H}^{+}$. Fig. 2 illustrates the initiation process in cationic polymerization. During the propagation step of the reaction, $\mathrm{H}^{+}$reacts with double bonds in the monomer (for example, vinyl ethers or epoxides). In radical type polymerization, cleavage of photoinitators during initiation generates radicals. The highly reactive radicals immediately attack double bonds in

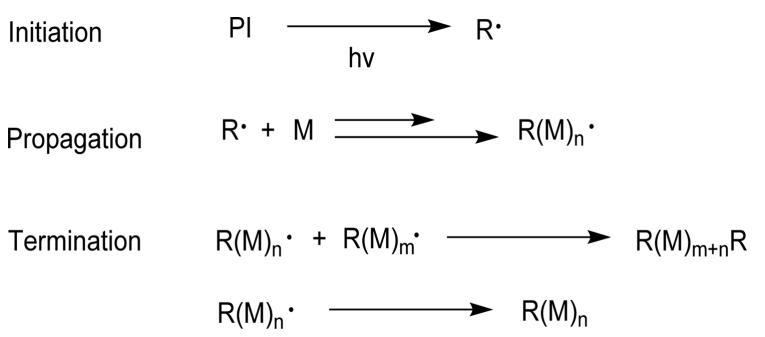

Fig. 1. Schematic illustration on initiation, propagation and termination of radical photopolymerization.

$$
\begin{array}{ll}
\text { Initiation } & \mathrm{Ar}_{3} \mathrm{~S}^{+} \mathrm{X}^{-} \underset{\mathrm{hv}}{ } \mathrm{Ar}^{+} \mathrm{X}^{-} \text {or } \mathrm{Ar}_{2} \mathrm{~S}^{+} \mathrm{X}^{-} \longrightarrow \mathrm{Ar}_{2} \mathrm{~S}+\mathrm{Ar}^{+} \mathrm{X}^{-} \text {or } \mathrm{Ar}+\mathrm{Ar}_{2} \mathrm{~S}^{+} \mathrm{X}^{-} \\
& \\
\text {Propagation } & \mathrm{H}^{+} \mathrm{X}^{-}+\mathrm{M} \longrightarrow \mathrm{H}^{+} \mathrm{X}^{-}+\text {additional products } \\
& \longrightarrow \mathrm{H}(\mathrm{M})_{\mathrm{n}}{ }^{+} \mathrm{X}^{-}
\end{array}
$$

Fig. 2. Schematic illustration on initiation and propagation of cationic photopolymerization (triarylsulfonium salt as an initiator). the monomer such as acrylates or methacrylates during polymerization. The radical polymerization is sensitive to oxygen present in the polymerizing media since radical can be captured by oxygen. In contrast, cationic polymerization is insensitive to oxygen content in the photocuring formulation. Another distinct difference between the two polymerization mechanisms is the polymerization termination step. During radical polymerization, radical-radical coupling is one of the major mechanisms of polymerization termination. However, cationic polymerization has living character which means polymerization can propagate even after light turned-off, due to unavailable coupling between cations. Often for photo-induced 3D printing processes like stereolithography (STL) and digital light processing (DLP), fine build structures are desired, and a high degree of control over the polymerization process is required. Therefore, cationic polymerization is not favored for these processes due to the aspect that cationic polymerization based photoinduced printing processes potentially have degraded resolution as a result of the living nature of the polymerization reaction.

\subsection{Photocurable monomer}

Among photocurable monomers in radical photopolymerization, acrylate functionalized monomers and telechelic oligomers have been most widely used due to rapid polymerization rate and wide range of available chemical structures. ${ }^{15,16)}$ There are several types of acrylated monomers with different characteristics. Cured acrylated epoxy shows good adhesion, chemical resistance, and flexibility. Acrylated urethane exhibits abrasion resistance, toughness and flexibility when cured. Acrylated polyether forms good films but shows degradation under UV or sunlight. ${ }^{15)}$ Acrylated polyester enables the formation of hard, tough, solvent resistant films. Comonomer system can also be utilized for radical photopolymerization. For example, acrylate monomers with vinyl ether monomers undergo photopolymerization to form copolymers. Under radical polymerization condition, vinyl ether monomers cannot proceed to form homopolymers. Therefore, when vinyl ether monomers are applied with acrylated monomers, the vinyl ether group is isolated in the polymer backbone from the acrylate group.

On top of the chemical structure of photocurable monomers, the number of functional groups in the photocurable monomer crucially affects the property of cured structures. There are many commercially available mono-, di-, tri-, and tetra- functionalized monomers shown in Fig. 3. When mono-functionalized monomers are solely applied for the photocurable formulation, resulting cured bodies are composed with entangled linear polymers. If multifunctional monomers are used in the formulation, cross-linked polymer networks are generated which makes cured body relatively stiff and mechanically tough. Based on the target property of cured body, multifunctional and mono-functional monomers may be utilized together in photocurable ink formulation. 
<smiles>C=CC(=O)OC1CC2CCC1(C)C2(C)C</smiles>

Isobornyl acrylate (monofunctional)<smiles>C=CC(=O)OCC(CC)(COC(=O)C=C)COC(=O)C=C</smiles>

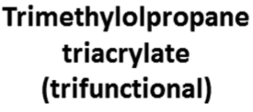<smiles>C=CC(=O)OCCCCCCOC(=O)C=C</smiles>

1,6-Hexanediol diacrylate (difunctional)<smiles>C=CC(=O)OCC(COC(=O)C=C)(COC(=O)C=C)COC(=O)C=C</smiles>

Pentaerythritol tetraacrylate (tetrafunctional)
Fig. 3. Photocurable arcylate monomers with different number of functional group.

\subsection{Ceramic suspension and Viscosity}

To create high quality, dense ceramics, the ceramic powder loading in green bodies must be high. Current research on rotational rheometer and vibrational viscometers allows for more accurate measurement of high solid loading, high viscosity slurries, which are optimal in ceramic processing as ceramic green bodies with low ceramic loading suffer from excessive shrinkage during binder burn-out. ${ }^{17)}$ Ceramic green bodies with low ceramic loading suffer from excessive shrinkage during binder burn-out. As ceramic solid loading is increased, the suspension viscosity increases as well. For optimum processing time and complex geometries, ceramic monomer suspensions for ceramic stereolithography (CerSLA) must be highly mobile fluid, which usually corresponds to a ceramic loading of less than $50 \mathrm{vol} \%$.

Viscosity upper limits for developing well defined, complex layers limit the ceramic loading that can be used when

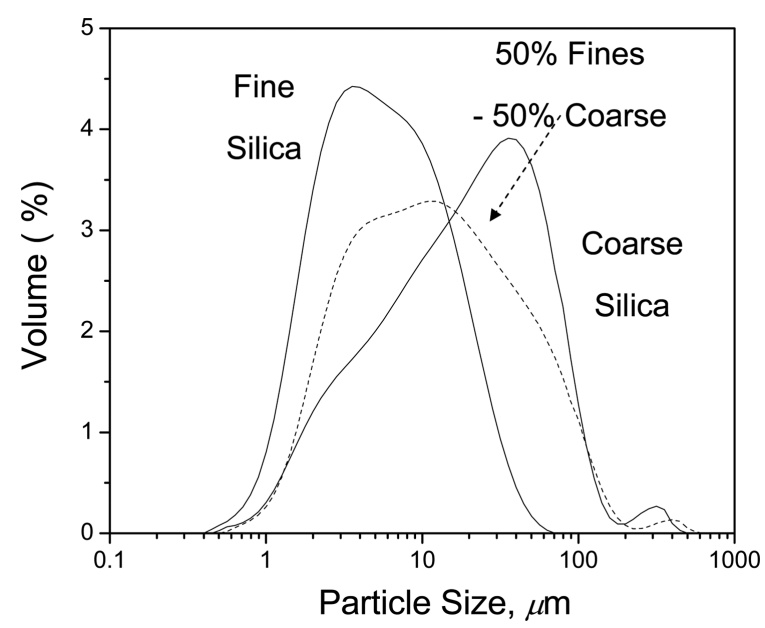

Fig. 4. Volume percent versus particle size distribution (PSD) of coarse and fine powders used to decrease the high viscosity of a 60 vol\% coarse $\mathrm{SiO}_{2}$ particles dispersed in the hexane diol diacrylate (HDDA) (from $\mathrm{Bae}^{29}$ ).

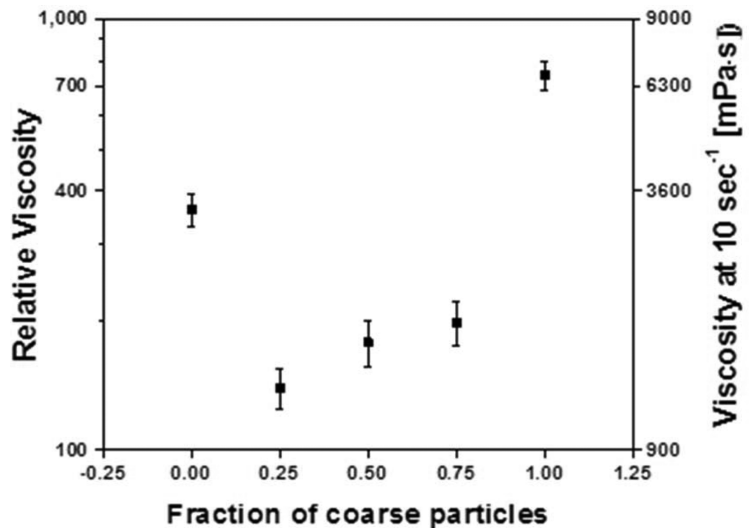

Fig. 5. Effect of particle size distribution on the relative viscosity of suspension. 60 volume percentage of silica dispersed suspensions are prepared by two different particle sizes; coarse $\left(\mathrm{d}_{50}: 26 \mu \mathrm{m}\right)$ and fine $\left(\mathrm{d}_{50}: 7 \mu \mathrm{m}\right)$ fused silica suspensions (from $\mathrm{Bae}^{29}$ ).

using ceramic stereolithography (CerSLA). The viscosity upper limit for a processable ink is generally around $3 \mathrm{~Pa}-\mathrm{s}$ with shear rates of $10 \mathrm{~s}^{-1}$. Relative viscosities for mono and bi-modal ceramic particle size distributions (PSDs) are shown in Fig. 4 and 5, for a constant solid loading of 60 vol\%. 100\% "coarse" ( $\mathrm{d}_{50} \approx 27$ microns) or "fine" silica powder $\left(\mathrm{d}_{50} \approx 7\right.$ microns) loaded suspensions have viscosities of 6.3 or $3.6 \mathrm{~Pa}$-s with shear rates of $10 \mathrm{~s}^{-1}$, respectively. $100 \%$ loaded coarse particle suspensions exceed the viscosity upper limit for processing. Reducing ceramic loading content minimally to $75 \mathrm{wt} \%$ fine and $25 \mathrm{wt} \%$ coarse powders abruptly decreased viscosity to $1.2 \mathrm{~Pa}$-s, to create a processable ceramic suspension.

There are two limiting cases for suspensions: 1) flocculated suspensions and 2) dispersed suspensions. Particles aggregate into floccus in flocculated suspensions, where floccus are behaving a shear-thinning during flow. As increasing flow rates further disrupt flocs, further flow of the suspension becomes easier. The behavior of flocculated suspensions is generally dominated by colloidal forces. In contrast, hydrodynamic effects limit the flow of dispersed suspensions and their behavior is well described by the Einstein equation.

For high volume fractions $\phi$, the Krieger-Dougherty (K-D) Equation is a more accurate model for Newtonian hydrodynamic viscosity ${ }^{18)}$ The relative viscosity, $\eta_{\gamma}$, is the viscosity of the loaded suspension divided by the viscosity of the liquid, $\eta_{0}$. The relative viscosity can also be expressed in terms of the K-D limit, $\phi_{0}$, where particles are "jammed".:

$$
\eta_{r}=\frac{\eta(\phi)}{\eta_{0}}=\left(1-\frac{\phi}{\phi_{0}}\right)^{-n}
$$

For an ideal suspension, the K-D limit is maximum particle packing fraction, $\phi_{0} \sim \phi_{\mathrm{m}}$, and the K-D exponent $\mathrm{n}$ is $2.5 \phi_{0}$. According to Eq. (1), viscosity abruptly increases as solid loading approaches the maximum volume or packing fraction, $\phi_{\mathrm{m}}$. The maximum packing fraction $\phi_{\mathrm{m}}$ depends on 
particle size distribution. ${ }^{19,20)}$

The Farris effect describes viscosity dependence on PSD for dispersed particle suspensions in the hydrodynamic limit, where $\mathrm{V}_{0}$ is the volume of suspended liquid, $\mathrm{V}_{1}$ the volume of fine powder, and $\mathrm{V}_{2}$ the volume of coarse particles in the suspension.

$$
\phi_{1}=\frac{V_{1}}{V_{0}+V_{1}} \quad \phi_{2}=\frac{V_{2}}{V_{0}+V_{1}+V_{2}}
$$

The total concentration, $\phi_{\mathrm{m}}$ and the relative viscosity of the suspension $\eta_{\gamma}$, can be expressed as

$$
\begin{aligned}
& \phi_{m}=\phi_{1}+\phi_{2}-\phi_{1} \phi_{2}=\phi_{2} \text { if } \phi_{1}=0 \\
& \eta_{r}=H\left(\phi_{1}\right) H\left(\phi_{2}\right)=H\left(\phi_{2}\right) \text { since } H\left(\phi_{1}\right)=1 \text { if } \phi_{1}=0
\end{aligned}
$$

where $\phi_{1}$ is the effective volume concentration of the fine particles, and $\phi_{2}$ the effective volume concentration of the coarse particles. The functions $H\left(\phi_{1}\right)$ and $H\left(\phi_{2}\right)$ may be determined by theoretical modeling or experiments. Given Eq. (4) along with the volume fractions of fine and coarse powders, Farris ${ }^{21}$ calculated the relative viscosities for bimodal systems versus blend ratio for a number of concentrations shown in Fig. 6. Modifying the PSD from a mono-modal to bimodal distribution decreases the relative viscosity of original suspension from 1000 to 15 (point $\mathrm{P}$ to $\mathrm{Q}$ ), and allows for the solid loading to be increased from 60 to $75 \%$ without increasing the viscosity (point $\mathrm{P}$ to $\mathrm{S}$ ). For high solid loaded suspensions (50\% or higher), PSD effects on the viscosity may be exploited to produce higher density green bodies and improve ceramic sinterability.

The tap density for different mixtures of fine and coarse powders is shown in Fig. 7. Tap density is larger for bimodal distributions compared to monomodal distributions. For a given $\phi$, according to Eq. (1), increasing the maximum

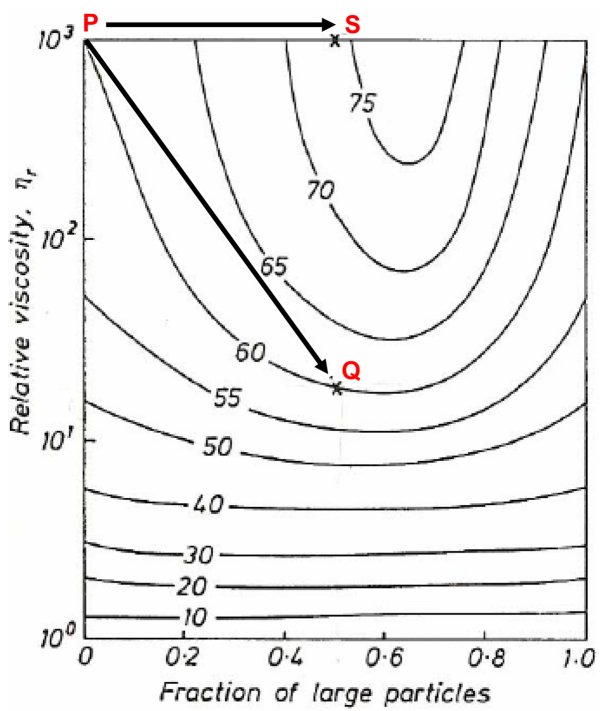

Fig. 6. Dramatically decreased relative viscosity can be explained by the Farris effect; Line $\mathrm{PQ}$ exhibits a $50 \%$ reduction in viscosity at the same solids loading. Line PS: An increase in the solids loading, from 60 to $75 \%$, without an increase in viscosity (from $\mathrm{Bae}^{29}$ ).

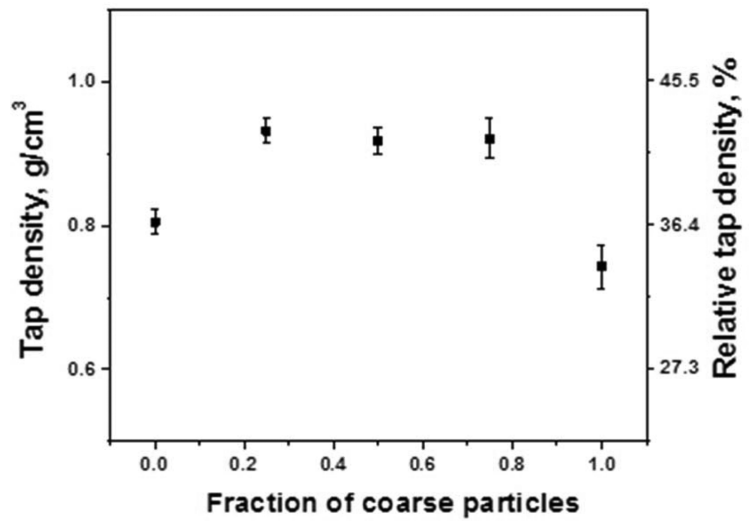

Fig. 7. Effect of particle size distribution on the tap density obtained with the mixture of two different particle sizes; coarse $\left(d_{50}: 26 \mu \mathrm{m}\right)$ and fine $\left(d_{50}: 7 \mu \mathrm{m}\right)$ fused silica suspensions (from $\mathrm{Bae}^{29}$ ).

volume fraction, $\phi_{\mathrm{m}}$, decreases the viscosity. Using bimodal PSDs in processing reduces the relative viscosity for the same percentage of ceramic solid loading as shown in Fig. 5.

\section{UV Curing Based Ceramic 3D Printing}

\subsection{Scattering, Cure depth, and Broadening}

In Ceramic stereolithography (CerSLA), a liquid bed of monomer, photoinitiator and ceramic powder is selectively polymerized with a UV laser, layer by layer, to form a ceramic green body. CAD files direct the laser writing. The polymerization reaction governing the writing of each slice requires a certain energy dose, $\mathrm{E}\left(\mathrm{J} / \mathrm{cm}^{2}\right)$. The time required to write a layer, $t_{w r i t e}$, is affected by the laser velocity, resin sensitivity $\left(\mathrm{D}_{\mathrm{p}}\right)$, and the critical energy dose $\left(\mathrm{E}_{\mathrm{c}}\right) . D_{p}$ is a material-dependent and wavelength-dependent related to the resin "penetration depth" for the laser wavelength. Jacob's version of the Beer-Lambert law ${ }^{21)}$ shows the relationship between the absolute cure depth, $C_{d}$, the resin sensitivity $\left(D_{p}\right)$, the energy dose $(\mathrm{E})$, and the critical energy dose $\left(E_{c}\right)$.

$$
C_{d}=D_{p} \ln \left(\frac{E}{E_{c}}\right)
$$

The resin sensitivity for a given ink formulation is dependent on the solid loading, ceramic particle size, and the refractive indices of the monomer and ceramic powder. The ceramic particles in the slurry scatter light. Empirically, it has been found that scattering increases with higher refractive index contrast, or higher $\Delta n=n_{\text {ceramic }}-n_{0}$. Griffith derived an approximation for the dependency of the resin sensitivity $\left[D_{p}\right]$ on the volume fraction $[\phi]$ in the limit where scattering dominates ${ }^{22,23)}$

$$
\begin{aligned}
& D_{p}=\frac{2}{3} \frac{d}{Q} \frac{1}{\phi}\left(\frac{n_{0}}{\Delta n}\right)^{2} \\
& D_{p}=\frac{2}{3} \frac{d}{Q} \frac{1}{\phi}\left(\frac{n_{0}}{\Delta n}\right)^{2}=\gamma \frac{d}{\phi}
\end{aligned}
$$


where $d$ is particle diameter, $\Delta n^{2}$ is the square of the refractive index difference between the ceramic and the monomer $\left[\Delta n^{2}=\left(n_{c}-n_{0}\right)^{2}\right], f$ the volume fraction solids, and $Q$ is the scattering efficiency term. For hexanediol diacrylate (HDDA) based systems, alumina $\left(n_{\mathrm{Al}_{2} \mathrm{O}_{3}}=1.72\right)$ and silica $\left(n_{\mathrm{SiO}_{2}}=1.56\right)$ suspensions show larger sensitivity compared to silicon nitride $\left(n_{\mathrm{Si}_{3} \mathrm{~N}_{4}}=2.1\right)$ suspensions due to lower refractive index contrast. ${ }^{22,23)}$ When designing a print system for a particular ceramic material with high refractive index, $n_{\text {ceramic }}$, the resin sensitivity will be strongly dependent upon the refractive index of the photocurable monomer, $n_{0}$, shown in Eq. (6). On the other hand, the case of the lower contrast of the refractive index the particle size and solid loading will dominate resin sensitivity. Eq. (7) shows the resin sensitivity as a function of particle size of ceramic powder and the solid volume fraction.

\subsection{Simulation}

Ideally when printing any material using stereolithography, the printed pixel matches in shape the beam profile. In ceramic stereolithography, regions of a ceramic powder, polymer monomer, and initiator slurry are selectively illuminated. The UV light activates photoinitiators, which create free radicals and initiate polymerization. Increased illumination increases the extent of polymerization until the polymer gel point. The printed region deviates from the beam profile due to 1) absorption and 2) scattering. The cure depth is the maximal depth at which the polymer receives sufficient illumination to reach gel point. ${ }^{24)}$ Eq. (5) in the previous section shows the relationship between the cure depth, $\mathrm{C}_{\mathrm{d}}$, the applied energy dose, E, the critical energy dose to achieve gel point, $\mathrm{E}_{\mathrm{c}}$, and $\mathrm{D}_{\mathrm{p}}$ an attenuation length describing the light signal decay in the print bed.

Predicting this attenuation length for high ceramic loading slurries is complicated. Garg et al. established a diffusion model for describing "light propagation in highly scattering and absorbing media". Gentry and Halloran examined scattering data for ceramic, monomer, initiator suspensions within the context of this diffusion model and found that refractive index contrast affects both the cure depth and the broadening (or the difference in the observed profile between the beam profile). ${ }^{25)}$ Gentry and Halloran found that when the refractive index of the ceramic powder closely matched the monomer refractive index both the cure depth and broadening were improved. ${ }^{25)}$

Inspired by Halloran and Gentry's observation that reduced refractive index contrast between the monomer slurry and the ceramic powder, our team is exploring the use of monomer refractive index matched ceramic coatings of the ceramic powder to improve cure depth and broadening. ${ }^{23,26-28)}$ We are using both experimental and computational methods to investigate this new approach to achieving high quality high solid loading ceramic printing of high refractive index ceramics. Since, our question is primarily whether coating can reduce scattering, which will in turn improve broadening and cure depth, we are not attempting to model perfectly cure depth or broadening. Our simulations are based on simple Mie Scattering models and aim to answer the question whether the coated ceramic particles are less of a scattering center than the uncoated scattering center. We correlate broadening with scattered light radial to the particle, while we correlate cure depth with all light propagating parallel to the print direction. Our preliminary results shown in Fig. 8 indicate that coating $0.5 \mu \mathrm{m}, 0.7 \mu \mathrm{m}$, $0.9 \mu \mathrm{m}$ radius silicon nitride particles with refractive index coatings of $\mathrm{n}=1.45,1.5,1.55,1.6,1.65,1.7,1.763,1.8,1.85$, and 1.9 with even thicknesses of $1 \mathrm{~nm}, 5 \mathrm{~nm}, 10 \mathrm{~nm}, 30 \mathrm{~nm}$, $50 \mathrm{~nm}, 75 \mathrm{~nm}$, and $100 \mathrm{~nm}$ show reduced scattered signal radially and therefore we expect they will show less broadening. Cure Depth results for the preliminary simulations are so far inconclusive.

\subsection{Build styles for minimizing internal stress}

In Ceramic sterolithograohy (CerSLA), CAD files are used to draw and slice an object into thin layers which are built in a resin bed by a UV laser, which selectively crosslinks layers to form a green body. The manner, in which each layer is built rather the $\mathrm{X}$ and $\mathrm{Y}$ controls for the UV (a)

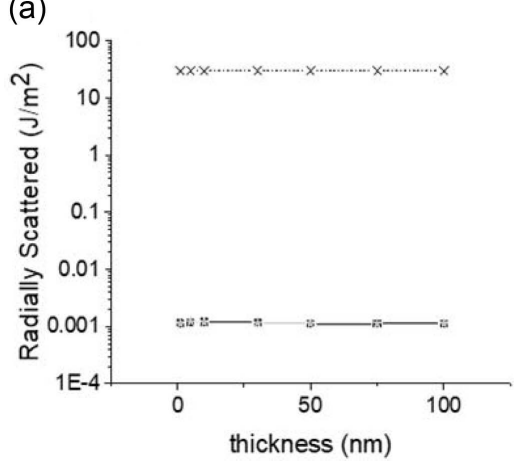

(b)

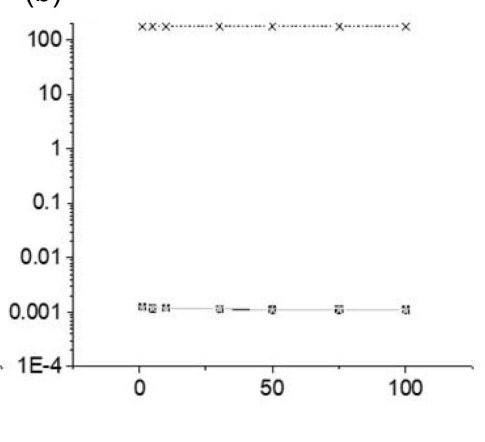

(c)

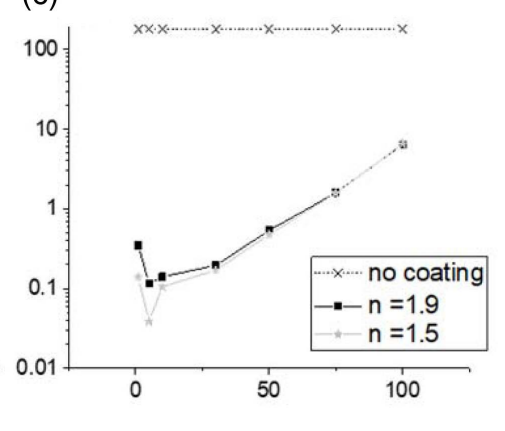

Fig. 8. Radially scattered intensities observed for Mie scattering codes (a) $0.5 \mu \mathrm{m}$, (b) $0.7 \mu \mathrm{m}$, and (c) $0.9 \mu \mathrm{m}$ radius of $\mathrm{Si}_{3} \mathrm{~N}_{4}$ particles coated with dielectrics of $n=1.5$ and $n=1.9$ of varying thickness. The calculated data for $n=1.45, n=1.55, n=1.6$, $\mathrm{n}=1.65, \mathrm{n}=1.7, \mathrm{n}=1.763, \mathrm{n}=1.8$, and $\mathrm{n}=.185$ coatings showed a similar trend We observe less radially scattering from the coated particles as compared to the uncoated particles shown in the dotted line. This suggests that less broadening is expected from coated ceramic particles. 
(a)

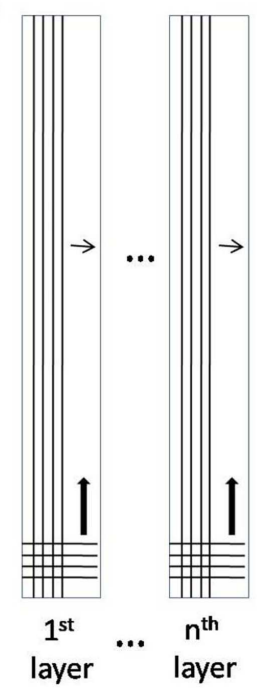

(b)

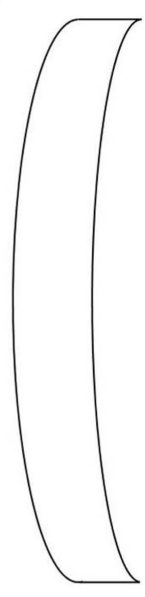

Fig. 9. Short vectors drawn using the WEAVE style. (a) cross-sectional view of each layer and (b) a distorted thin vertical wall attributed to the net result of subsequent shrinkage at the regions which largely attached to the lower layer and the border lines (from $\mathrm{Bae}^{29}$ ).

laser, is the "build style". Incorrect choice of build style creates defects, distortions, and problems with delamination in the green body. Most distortions originate due to volume shrinkage. CerSLA is derived from stereolithography (SL), for which there are multiple "build styles" to prevent shrinkage.

Conventional "WEAVE build style" developed by Jacobs cures the outside of a layer or "border lines" first followed by inside "fill lines" in a hatch pattern. ${ }^{14)}$ Fill lines can connect two border lines, or attach to just one border line leaving a small gap between the other lines ("end contraction"). Weave hatches generally utilize a 0.011 " spacing for both the $\mathrm{x}$ and $\mathrm{y}$ hatches. WEAVE style reduces the residual monomer present in the cured part. ${ }^{29)}$ WEAVE build style results in less uncured monomer in the built part, but creates parts with large solidified regions that cause distortions. Internal stresses concentrate in the part during builds. As shown in Fig. 9, shrinkage of short X-hatches resulted in a "wave" of curing. Fig. 9(b) shows a distorted thin vertical wall not drawn to scale. Curvature in the built part is due to differential hatch shrinkage.

Newly developed build styles seek to reduce distortions by avoiding internal stress accumulation during build. The most significant distortions are incurred in large, flat, and complex 3D objects. "STaggered weave build style" enables internal stress relief during builds by minimizing the contact area between build lines. Fig. 9 shows the conventional WEAVE style and STaggered hatch styles. During WEAVE style builds internal stresses incurred during the build result in the majority of significant distortions, while STaggered hatch build styles incur less internal stress between layers by staggering hatches to prevent long cracks from

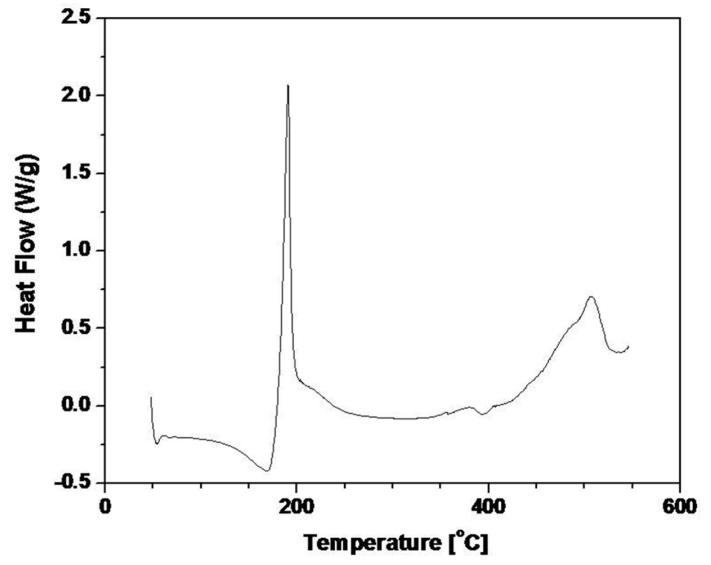

Fig. 10. Heat flow as a function of temperature measured by DSC. Exothermic heat flow around $200^{\circ} \mathrm{C}$ represents thermal polymerization induced by thermal decomposition of photoinitiator (from $\mathrm{Bae}^{29}$ ).

forming perpendicular to the layers.

With green bodies built at less internal stresses of STaggered build style, a binder burn out step and other post-processing steps are followed. During binder burnout, polymer is removed and the ceramic is densified. In addition to the flaws incurred during the build process, flaws are created during the binder burn out process and other additional post-processing steps. Simple cube shaped green specimens may be used to determine the temperature at which cracks initiate during heating and the BBO process. ${ }^{30)}$ This example shows that while the most significant stresses are associated with mass loss during $\mathrm{BBO}$, cracking may occur before mass loss is observed. TGA data is useful but not always sufficient for designing optimal BBO conditions to minimize cracking.

Differential Scanning Calorimeter (DSC) is used to determine chemical events other than pyrolysis during the BBO process that can lead to internal stress development in the ceramic. Fig. 10 is a DSC scan for the HDDA-EPETA monomer mixture with the hydroxyketone photoinitiator before photopolymerization, the polymer system used for the silica example given in this review article. Thermal decomposition of the ketone occurs as an endothermic event around $170^{\circ} \mathrm{C}$, with a large exothermic effect between $180-200^{\circ} \mathrm{C}$ from the exothermic polymerization of the acrylates. Thus the ketone photoinitiator serves as a thermal initiator if heated to $\sim 200^{\circ} \mathrm{C}$. In this specific example, it shows that varying build styles such as different hatch spacing and no end retracts leaves or removes residual monomer in the green bodies, alleviating internal stress and preventing cracking during $\mathrm{BBO} .{ }^{31)}$

\section{Application}

3D printing of ceramic has the ability to revolutionize many industries. We will focus on two areas: 1) battery and 2) ceramic mold fabrication. $3 \mathrm{D}$ printing has three major 
advantages compared to more conventional fabrication methods: 1) it allows for rapid fabrication, 2) it allows for the production of complicated geometries that can be difficult to fabricate otherwise, and 3) it allows for more ready customization of geometry.

Investment casting is often used for production of industrial parts like jet engines, car parts. ${ }^{3,30)}$ The investment cast molds are generally made of ceramics like silica due to their hardness, toughness, and thermal expansion properties. Traditional tooling to build ceramic molds are time consuming, expansive, and often do not allow for fine features. Using CerSLA to produce ceramic molds allows for the production of finer and more complex structures. These structures can be used to produce lightweight, but strong parts or to direct heat flow in the part. Additionally, since CerSLA fabrication is rapid compared to conventional fabrication means, molds can be fabricated, tested, and tweaked in rapid succession. The mold editing process is also aided by the fact that CerSLA like other 3D printing methods uses $\mathrm{CAD}$, or digital blueprints. Bae et al. showed that CerSLA could be used to produce superior silica molds for Turbine Airfoils that had finer features than are possible to produce with conventional means and improved thickness consistency. ${ }^{3,30,31)}$

Batteries are complex devices with multiple components, each of which has different materials properties. For batteries there is a tradeoff between energy and power. Improving both energy and power in batteries requires the ability to design ion transport in the device. In order to design ion transport in the device, the device structure must be optimally engineered in all parts of the device (anode, cathode, separator, current collector, etc). In order to engineer ion transport in batteries, the interfaces between layers of the material should be mechanically strong and not hamper ion transport. Additionally, the microstructure and architecture of each part of the components of battery should be wellsuited to ion transport. Since 3D printing methods are being developed on the micron scale fabrication (and for specific polymers on the nano-scale fabrication), 3D printing can be used to create transport directing structures in battery materials. ${ }^{32)}$ Also, as 3D printing methods exist for polymers, ceramics, and metals, it is possible to envision printing each component of the battery, which ideally should be beneficial to interfacial properties of the device. In our lab, we are currently developing printing processes for designing new, engineered cathode and current collector materials. We soon hope to begin work on printing anode materials as well.

We have provided two examples where we see an immediate potential for 3D printing to change industry fabrication. $3 \mathrm{D}$ printing is already revolutionizing biomedical technologies (i.e producing improved hip replacements, bone scaffolds, tissue mimics, etc). Also, 3D printing provides advantages over existing fabrication methods in all areas where rapid production of individual and highly structured objects is desired.

\section{Acknowledgements}

This research was supported by the Office of Naval Research under Grant (N00421-06-1-0002), the Korean Institute of Materials Science (KIMS) under the Korea Institute of Machinery \& Materials, and the Korea Institute of Energy Research (KIER). (PNK5280, PICL140).

\section{REFERENCES}

1. K. Upadhya, J. M. Yang, and W. P. Hoffmann, "Materials for Ultrahigh Temperature Structural Applications," Am. Ceram. Soc. Bull., 76 [12] 51-6 (1997).

2. H. Park, H. W. Kim, and H.-E. Kim, "Oxidation and Strength Retention of Monolithic $\mathrm{Si}_{3} \mathrm{~N}_{4}$ and Nanocomposite $\mathrm{Si}_{3} \mathrm{~N}_{4}$-SiC with $\mathrm{Yb}_{2} \mathrm{O}_{3}$ as a Sintering Additive," J. Am. Ceram. Soc., 81 [8] 2130-34 (1989).

3. C.-J. Bae and J. W. Halloran, "Integrally Cored Ceramic Mold Fabricated by Ceramic Stereolithography," Int. J. Appl. Ceram. Technol., 8 [6] 1255-62 (2011).

4. H. Huang, "Machining Characteristics and Surface Integrity of Yttria Stabilized Tetragonal Zirconia in High Speed Deep Griding," Mater. Sci. Eng., A, 345 [1-2] 155-63 (2003).

5. M. Guazzato, K. Proos, G. Sara, and M. V. Swain, "Strength, Reliability, and Mode of Fracture of Bilayered Porcelain/ Core Ceramics," Int. J. Prosthodontics, 17 [2] 142-49 (2004).

6. A. Zocca, P. Colombo, C. M. Gomes, and J. Gunster, "Additive Manufacturing of Ceramics: Issues, Potentialities, and Opportunities," J. Am. Ceram. Soc., 98 [7] 1983-2001 (2015).

7. J. Moon, A. C. Caballero, L. Hozer, Y. M. Chiang, and M. J. Cima, "Fabrication of Functionally Graded Reaction Infiltrated SiC-Si Composite by Three-Dimensional Printing (3DP $\left.{ }^{\mathrm{TM}}\right)$ Process," Mater. Sci. Eng., A, 298 [1-2] 110-19 (2001).

8. S. Das, M. Wohlert, J. J. Beaman, and D. L. Bourell, "Producing Metal Parts with Selective Laser Sintering/Hot Isostatic Pressing," JOM, 50 [12] 17-20 (1998).

9. T. Friedel, N. Travitzky, F. Niebling, M. Scheffler, and P. Greil, "Fabrication of Polymer Derived Ceramic Parts by Selective Laser Curing," J. Eur. Ceram. Soc., 25 [2-3] 19397 (2005).

10. J. Deckers, J. Vleugels, and J.-P. Kruth, "Additive Manufacturing of Ceramics: A Review," J. Ceram. Sci. Tech., $\mathbf{5}$ [4] 245-60 (2014).

11. K. Subramanian, N. Vail, J. Barlow, and H. Marcus, "Selective Laser Sintering of Alumina with Polymer Binders," Rapid Prototyping J., 1 [2] 24-35 (1995).

12. M. L. Griffith and J. W. Halloran, "Freeform Fabrication of Ceramics via Stereolithography," J. Am. Ceram. Soc., 79 [10] 2601-8 (1996).

13. L. J. Hornbeck, "Digital Light Processing ${ }^{\mathrm{TM}}$ for HighBrightness High-Resolution Applications," Proc. SPIE, 3013 1-14 (1997).

14. P. F. Jacobs, Rapid Prototyping \& Manufacturing-Fundamentals of Stereolithography; pp. 397, SME, California, 1992.

15. C. E. Hoyle, "Photocurable Coatings," Radiat. Curing Polym. 
Mater., 417 1-16 (1990).

16. C. Decker, "UV-Radiation Curing Chemistry," Pigm. Resin Technol., 30 [5] 278-86 (2001).

17. H. Ji and H. S. Lee, "Comparison of the Viscosity of Ceramic Slurries Using a Rotational Rheometerand a Vibrational Viscometer," J. Korean Ceram. Soc., 49 [6] 542-48 (2012).

18. I. M. Krieger and T. J. Dougherty, "A Mechanism for NonNewtonian Flow in Suspensions of Rigid Spheres," Trans. Soc. Rheol., 3 137-52 (1959).

19. C. C. Furnas, "Grading Aggregates: I, Mathematical Relations for Beds of Broken Solids of Maximum Density," Ind. Eng. Chem., 23 [9] 1052-58 (1931).

20. R. K. Mcgeary, "Mechanical Packing of Spherical Particles," J. Am. Ceram. Soc., 44 [10] 513-22 (1961).

21. R. J. Farris, "Prediction of the Viscosity of Multimodal Suspensions from Unimodal Viscosity Data," Trans. Soc. Rheol., 12 [2] 281-301 (1968).

22. M. L. Griffith, Stereolithography of Ceramics, in Ph.D. Thesis, University of Michigan, Ann Arbor, 1995.

23. M. L. Griffith and J. W. Halloran, "Scattering of Ultraviolet Radiation in Turbid Suspensions," J. Appl. Phys., 81 [6] 2538-46 (1997).

24. J. W. Halloran, "Ceramic Stereolithography: Additive Manufacturing for Ceramics by Photopolymerization," Annu. Rev. Mater. Res., 46 19-40 (2016).

25. S. P. Gentry and J. W. Halloran, "Depth and Width of Cured Lines in Photopolymerizable Ceramic Suspen- sions," J. Eur. Ceram. Soc., 33 [10] 1981-88 (2013).

26. P. Yang, B. C. Gao, W. J. Wiscombe, M. I. Mishchenko, S. Platnick, H. L. Huang, B. A. Baum, Y. X. Hu, D. Winker, S. C. Tsay, and S. K. Park, "Inherent and Apparent Scattering Properties of Coated or Uncoated Spheres Embedded in an Absorbing Host Medium," Appl. Opt., 41 [15] 2740-59 (2002).

27. C. F. Bohren and D. M. Huffman, "Absorption and Scattering by a Sphere," pp. 82-129 in Absorption and Scattering of Light by Small Particles, Wiley, 1983.

28. I. W. Sudiarta and P. Chylek, "Mie-Scattering Formalism for Spherical Particles Embedded in an Absorbing Medium," J. Opt. Soc. Am. A. Opt. Image Sci. Vis., 18 [6] 1275-78 (2001).

29. S. O. Onuh and K. K. B. Hon, "Application of the Taguchi Method and New Hatch Styles for Quality Improvement in Stereolithography," Proc. Inst. Mech. Eng., Part B, 212 [6] 461-71 (1998).

30. C.-J. Bae, Integrally Cored Ceramic Investment Casting Mold Fabricated by Ceramic Stereolithography, in Ph.D. Thesis, University of Michigan, Ann Arbor, 2008.

31. C.-J. Bae and J. W. Halloran, "Integrally Cored Ceramic Mold Fabricated by Ceramic Stereolithography," Int. J. Appl. Ceram. Technol., 8 [6] 1289-95 (2011).

32. C.-J. Bae, C. K. Erdonmez, J. W. Halloran, Y.-M. Chiang, "Design of Battery Electrodes with Dual-Scale Porosity to Minimize Tortuosity and Maximize Performance," Adv. Mater., 25 [9] 1254-58 (2013). 